\title{
Desempenho agronômico de cultivares de café resistentes à ferrugem no Estado de Minas Gerais, Brasil
}

\author{
Alex Mendonça de Carvalho ('); Antônio Nazareno Guimarães Mendes ('); Cesar Elias Botelho (²); \\ Antonio Carlos Baião de Oliveira ( $\left.{ }^{3,4}\right)$; Juliana Costa de Rezende $\left({ }^{1 *}\right)$; Ramiro Machado Rezende $\left({ }^{1}\right)$ \\ (') Universidade Federal de Lavras (UFLA), Departamento de Agricultura, 37200-000 Lavras (MG), Brasil. \\ (2) Empresa de Pesquisa Agropecuária de Minas Gerais (Epamig), Caixa Postal 176, 37200-000 Lavras (MG), Brasil. \\ (3) Embrapa Café, 70770-901 Brasília (DF), Brasil. \\ (4) Epamig, Caixa Postal 216, 36570-000 Viçosa (MG), Brasil. \\ (*) Autora correspondente: julianacr@epamig.ufla.br
}

Recebido: 3/abr./2012; Aceito: 7/dez./2012

\begin{abstract}
Resumo
Objetivou-se no trabalho avaliar o comportamento de 24 cultivares de cafeeiro, sendo 22 resistentes à ferrugem e duas testemunhas suscetíveis, em diferentes ambientes de Minas Gerais, a fim de contribuir para a recomendação técnica. Os experimentos foram instalados em quatro municípios (Lavras, Campos Altos, Patrocínio e Turmalina). O delineamento experimental utilizado foi o de blocos casualizados com três repetições, no espaçamento de 3,5x0,7 m e parcelas de dez plantas. As avaliações de produtividade, porcentagem de grãos classificados em peneira 16 acima e vigor vegetativo referem-se a quatro colheitas (2008 a 2012). Foram avaliadas, também, a adaptabilidade e estabilidade da produtividade das cultivares. As cultivares Sabiá Tardio, Pau Brasil MG1, Obatã IAC 1669-20, Catucaí Amarelo 24/137 e IPR 103 são promissoras para as referidas regiões cafeeiras. Essas cultivares aliam estabilidade e adaptabilidade independentemente do ambiente, com desempenho agronômico superior.
\end{abstract}

Palavras-chave: Coffea arabica, produtividade, adaptabilidade, estabilidade fenotípica.

\section{Agronomic performance of coffee cultivars resistant to coffee rust in Minas Gerais State, Brazil}

\begin{abstract}
The purpose of this study was to evaluate the agronomic performance of 24 coffee cultivars (22 rust resistant cultivars and 2 susceptible control varieties) in different environments of Minas Gerais State, Brazil. The coffee plants were planted in four locations (Lavras, Campos Altos, Patrocínio and Turmalina) in three agroclimatic regions. The experiments were arranged in randomized complete block design, with three replications in plots measuring $3.5 \times 0.7 \mathrm{~m}$, with 10 plants per plot. The following traits were analyzed: yield, percentage of grains retained in sieve size 16 and vegetative vigor from four cropping years (2008 to 2012) and the adaptability and stability in yield. The cultivars showed differential performance in the four environments. The cultivars Sabiá Tardio, Pau Brasil MG1, Obatã IAC 1669-20, Catucaí Amarelo 24/137 and IPR 103 were the most promising because they combined higher agronomic stability and adaptability in favorable and unfavorable environments.
\end{abstract}

Key words: Coffea arabica, yield, adaptability, fenotype stability.

\section{INTRODUÇÃO}

A pesquisa agrícola tem colocado inúmeras tecnologias à disposição dos produtores, com a finalidade de aumentar a produtividade e a renda do cafeicultor. No caso do melhoramento genético, diversas cultivares resistentes à ferrugem-alaranjada (Hemileia vastatrix Berk. et Br), principal doença da cultura, já foram liberadas para uso comercial. A resistência da planta minimiza o uso de produtos fitossanitários no manejo do cafeeiro, notadamente, fungicidas que, na maioria das vezes, são de alta toxicidade ao homem, animais domésticos e silvestres.
Apesar do elevado potencial produtivo e de outras características morfoagronômicas de interesse para o cafeicultor, essas novas cultivares vêm sendo adotadas em ritmo lento pelos produtores. É provável que a razão desse comportamento seja a falta de informaçóes sobre o comportamento agronômico de tais cultivares.

No Brasil, as regiōes cafeeiras são bem distintas em relação ao ambiente, fato que levará à resposta diferenciada (CucolotTo et al., 2007). Embora os melhoristas, em geral, interpretem essa interação como negativa, pois sugere uma barreira ao ganho de seleção, VASCONCELOS et al. (2010) ressaltam que as interaçóes 
significativas representam uma oportunidade de exploração. A adaptação de genótipos a ambientes específicos pode fazer a diferença entre a boa e a excelente cultivar (CARgnin et al., 2006).

Em cafeeiro, alguns autores obtiveram informações destes efeitos positivos de interação pela aplicação de métodos estatísticos (Botelho et al., 2010; Cilas et al., 2011; Gichimu e Omondi, 2010). No método proposto por AnNicCHiarico (1992), a estabilidade é estimada pela superioridade de um genótipo em relaçáo ao ambiente. Este método é firmado no cálculo de um índice de recomendação ou de confiança, que indica a probabilidade de que o desempenho de um genótipo seja superior sobre os outros. Assim, é considerada ideal a cultivar que tiver o menor risco de ser adotada, pois possui o maior índice de confiança (Correa et al., 2006). Autores como Grunvald et al. (2008) e Condé et al. (2010) recomendam a utilização deste método pela facilidade da interpretação.

O objetivo desta pesquisa consiste em avaliar o desempenho agronômico, a adaptabilidade e estabilidade de cultivares de café arábica resistentes à ferrugem, a fim de auxiliar na recomendação regional.

\section{MATERIAL E MÉTODOS}

Os experimentos foram desenvolvidos de dezembro de 2005 a julho de 2011, nas três principais regiốes cafeeiras do Estado (Sul de Minas, Alto Paranaíba e Vale do Jequitinhonha), conforme tabela 1 . O experimento foi instalado em solos classificados como Latossolo Vermelho Distroférrico, visto que possuem saturação por bases baixa $(\mathrm{V}<50 \%)$ e teores de $\mathrm{Fe}_{2} \mathrm{O}_{3}$ (pelo $\mathrm{H}_{2} \mathrm{SO}_{4}$ ) de 180 a $360 \mathrm{~g} \mathrm{~kg}^{-1}$ nos primeiros $100 \mathrm{~cm}$ do horizonte B (SAntos et al., 2006)

As cultivares foram desenvolvidas pelos programas de melhoramento genético de Coffea arabica L. do IAC, EPAMIG/UFLA/UFV, IAPAR e MAPA/Fundação Procafé. Do total de 24 cultivares, foram escolhidas 22 resistentes à ferrugem (Catucaí Amarelo 2SL, Catucaí Amarelo 24/137, Catucaí Amarelo 20/15 cv 479, Catucaí 785/15, Catucaí Vermelho 20/15 cv 476, Sabiá Tardio, IBC Palma 2, Acauâ, Oeiras MG 6851, Catiguá MG 1, Sacramento MG 1, Catiguá MG 2, Araponga MG 1, Paraíso MG H419-1, Pau Brasil MG 1, Tupi IAC 1669-33, Obatã IAC 1669-20, Iapar 59, IPR 98,
IPR 99, IPR 103 e IPR 104) e duas suscetíveis (Topázio MG 1190 e Bourbon Amarelo IAC J10).

O delineamento experimental foi o de blocos casualizados, com três repetiçôes, parcelas de dez plantas, em que se considerou como parcela útil as seis plantas centrais. As cultivares foram implantadas em espaçamento de $3,5 \mathrm{~m}$ entre linhas e $0,70 \mathrm{~m}$ entre plantas. $\mathrm{O}$ ensaio instalado em Turmalina foi irrigado por gotejamento.

As características agronômicas avaliadas nas quatro primeiras colheitas (safras 2008/2009 a 2011/2012) foram: (1) produtividade (sacas de $60 \mathrm{~kg}$ de café beneficiado por ha $\left.{ }^{-1}\right)$; (2) classificação do café por peneira; (3) vigor vegetativo. A produtividade foi estimada assumindo que 480 litros de café equivalem a uma saca beneficiada (Carvalho et al., 2009); para classificação, utilizou-se uma amostra de 300 gramas que foi submetida ao conjunto de peneiras de 19/18/17 e 16 e para o vigor vegetativo, atribuíram-se notas conforme escala arbitrária de 10 pontos (Carvalho et al., 1979).

Para a produtividade, foi realizada análise de variância, no esquema conjunto de quatro locais e subdividida no tempo, cujo conjunto de duas colheitas (biênio) consistia a subparcela. Portanto, foram considerados dois biênios, objetivando reduzir o efeito da bienalidade da produção e melhorar a precisão experimental (BonOMO et al., 2004; BотеLho et al., 2010). Na análise conjunta das demais características agronômicas, considerou-se a média das quatro colheitas. As análises foram realizadas após a constatação da homogeneidade das variâncias, por meio do teste Hartley. Posteriormente, as médias foram comparadas pelo teste Scott Knott com nível de significância de $\mathrm{p}<0,01$ de probabilidade. Utilizou-se o aplicativo computacional Sisvar (Ferreira, 2011).

Após a constatação da significância da interação entre progênies, biênios e locais realizou-se a análise da adaptabilidade e estabilidade das cultivares, considerando o ambiente as combinaçóes de biênios com locais. Foi utilizado o método sugerido por AnNicChiarico (1992), o qual propõe a adoção de um índice de confiança (reliability index), que estima o risco da adoçáo de determinado genótipo. Os procedimentos para os cálculos pelo referido método foi realizado após a transformação das médias de produtividade de cada cultivar e ambiente, em porcentagem da média do ambiente. Posteriormente, estimou-se a média $Y_{\mathrm{i}(\mathrm{GFD})}$ e o desvio-padrão $\left(\mathrm{S}_{\mathrm{i}(\mathrm{GFD})}\right)$ das porcentagens de cada cultivar para os ambientes de maneira geral $(\mathrm{G})$, favoráveis $(\mathrm{F})$ e desfavoráveis $(\mathrm{D})$.

Tabela 1. Regiôes geográficas, variáveis climáticas e caracterizaçáo dos locais de instalação dos experimentos no Estado de Minas Gerais

\begin{tabular}{lcccc} 
Município & Lavras & Campos Altos & Patrocínio & Turmalina \\
\hline Região & Sul & Alto Paranaíba & Alto Paranaíba & Jequitinhonha \\
\hline Altitude $(\mathrm{m})$ & 919 & 1230 & 966 & 820 \\
\hline Temperatura média $\left({ }^{\circ} \mathrm{C}\right)$ & 19,3 & 17,6 & 22,0 & 21,3 \\
\hline Precipitação pluvial média anual $(\mathrm{mm})$ & 1529 & 1830 & 1620 & 1450 \\
Região cafeeira & Sul de Minas & Cerrado de Minas & Cerrado de Minas & Chapada de Minas \\
\hline
\end{tabular}


A partir dessas estimativas, foram obtidos os índices de confiança $\mathrm{I}_{\mathrm{i}(\mathrm{GFD})}$ geral $(\mathrm{G})$, favoráveis $(\mathrm{F})$ e desfavoráveis (D) para cada cultivar por meio do seguinte estimador:

$I_{i}=Y_{i}-Z(1-\alpha) \cdot S i$,

Em que:

$\mathrm{I}_{\mathrm{i}}$ : índice de confiança (\%); $\mathrm{Y}_{i}$ : média da cultivar i em porcentagem; $Z$ : valor na distribuição normal estandardizada no qual a função de distribuiçáo acumulada atinge o valor percentual $(1-\alpha)$ e Si: desvio-padrão dos valores percentuais. Quanto maior esse índice, menor o risco de adoção da cultivar. Para esta análise, foi utilizado o programa Estabilidade (Ferreira e Zambalde, 1997).

\section{RESULTADOS E DISCUSSÃO}

Houve efeito significativo para cultivares, locais, biênios e para as interaçôes entre cultivares e locais; locais e biênios; cultivares e biênios, e entre cultivares, locais e biênios. A significância da interaçáo demonstra que o comportamento das cultivares varia com os ambientes. Em relação ao local, observa-se que para o município de
Lavras foram obtidos quatro grupos de cultivares em relação às produtividades, com destaque para as cultivares Catucaí Amarelo 20/15 cv 479, Pau Brasil MG 1, Obatã IAC 1669-20 e IPR 99, com produtividade variando de 44,8 a 49,5 sacas ha-1 (Tabela 2).

Em Campos Altos destacam-se três grupos, nos quais as cultivares Sabiá Tardio, IBC Palma 2, Pau Brasil MG 1 e Topázio MG 1190 proporcionaram produtividade entre 37,5 e 41,7 sacas hä ${ }^{-1}$. Em Patrocínio distinguiram-se cinco grupos, com destaque para 'Sabiá Tardio' (56,9 sacas ha $\left.{ }^{-1}\right)$. Em Turmalina, a exemplo de Campos Altos, três grupos de cultivares diferenciaram-se em relação à produtividade. $\mathrm{O}$ grupo com produtividade maior foi formado por cinco cultivares: Catucaí Amarelo 2SL, Catucaí Amarelo 24/137, Sabiá Tardio, Obatã IAC 1669-20 e IPR 103, com produtividade variando entre 49,3 e 55,3 sacas $\mathrm{ha}^{-1}$.

Com base na média geral de quatro colheitas, a produtividade das cultivares Sabiá Tardio, Pau Brasil MG 1, Obatá IAC 1669-20, Catucaí Amarelo 24/137 e IPR 103 sobressaíram-se às demais cultivares (Tabela 2). O potencial produtivo dessas cultivares também foi relatado por Parva et al. (2010). Para estes autores, houve destaque para o comportamento agronômico e produtivo de 18 cultivares de porte baixo, no município de Varginha, especialmente para as cultivares Sabiá Tardio e Obată IAC 1669-20. Dias

Tabela 2. Médias de produtividade, em sacas de $60 \mathrm{~kg}$ de café beneficiado por hectare, de 24 cultivares comerciais de cafeeiro, avaliadas nas safras de 2008/2009 a 2011/2012, nos municípios de Lavras (LA), Campos Altos (CA), Patrocínio (PA) e Turmalina (TU) em Minas Gerais e média geral dos quatro ambientes

\begin{tabular}{|c|c|c|c|c|c|}
\hline Cultivares & LA & CA & PA & TU & Média \\
\hline Catucaí Amarelo 2SL & $32,7 \mathrm{cB}$ & $33,3 \mathrm{bB}$ & $44,3 \mathrm{cA}$ & 49,3 aA & 39,9 b \\
\hline Catucaí Amarelo 24/137 & $42,8 \mathrm{bB}$ & $31,7 \mathrm{bC}$ & $47,9 \mathrm{bB}$ & 55,3 aA & $44,4 \mathrm{a}$ \\
\hline Catucaí Amarelo 20/15 cv 479 & 44,8 aA & 30,2 bc & $36,4 \mathrm{~dB}$ & $46,5 \mathrm{bA}$ & $39,5 b$ \\
\hline Catucaí 785/15 & $23,1 \mathrm{eB}$ & $21,5 \mathrm{cB}$ & $22,6 \mathrm{eB}$ & $36,0 \mathrm{cA}$ & $25,8 \mathrm{~g}$ \\
\hline Catucaí Vermelho 20/15 cv 476 & $26,7 \mathrm{~dB}$ & $21,6 c C$ & $29,7 \mathrm{~dB}$ & $43,6 \mathrm{bA}$ & $30,4 \mathrm{f}$ \\
\hline Sabiá Tardio & $41,6 \mathrm{bB}$ & $37,5 \mathrm{aB}$ & 56,9 aA & $51,5 \mathrm{aA}$ & $46,9 \mathrm{a}$ \\
\hline IBC Palma 2 & $38,2 \mathrm{bA}$ & $38,4 \mathrm{aA}$ & $38,2 \mathrm{cA}$ & $43,9 \mathrm{bA}$ & 39,7 b \\
\hline Acauã & $32,4 \mathrm{cB}$ & $23,0 \mathrm{cC}$ & $44,7 \mathrm{cA}$ & 46,7 bA & $36,7 c$ \\
\hline Oeiras MG 6851 & $28,4 \mathrm{~dB}$ & $33,6 \mathrm{bB}$ & $29,8 \mathrm{~dB}$ & $42,2 \mathrm{bA}$ & $33,5 \mathrm{~d}$ \\
\hline Catiguá MG 1 & $35,5 \mathrm{cA}$ & $23,9 \mathrm{cB}$ & $31,7 \mathrm{dA}$ & $34,0 \mathrm{cA}$ & 31,3 e \\
\hline Sacramento MG 1 & $31,6 \mathrm{cB}$ & 30,2 bB & $39,9 \mathrm{cA}$ & $36,7 \mathrm{cA}$ & $34,6 d$ \\
\hline Catiguá MG 2 & $41,3 \mathrm{bA}$ & $31,0 \mathrm{bB}$ & $35,3 \mathrm{~dB}$ & $32,0 \mathrm{cB}$ & $34,9 d$ \\
\hline Araponga MG 1 & $31,1 \mathrm{cA}$ & $31,8 \mathrm{bA}$ & $33,6 \mathrm{dA}$ & $33,8 \mathrm{cA}$ & $32,6 \mathrm{e}$ \\
\hline Paraíso MG H419-1 & $29,5 \mathrm{dA}$ & $33,0 \mathrm{bA}$ & $31,0 \mathrm{dA}$ & $34,5 \mathrm{cA}$ & 32,0 e \\
\hline Pau Brasil MG 1 & $45,7 \mathrm{aA}$ & $38,8 \mathrm{aB}$ & $38,5 \mathrm{cB}$ & $46,3 \mathrm{bA}$ & $42,3 \mathrm{a}$ \\
\hline Tupi IAC 1669-33 & $36,0 \mathrm{cC}$ & $24,2 \mathrm{cD}$ & $48,2 \mathrm{bA}$ & 41,9 bB & $37,6 \mathrm{c}$ \\
\hline Obatã IAC 1669-20 & 49,5 aA & $31,4 \mathrm{bB}$ & $46,0 \mathrm{bA}$ & $53,1 \mathrm{aA}$ & $45,0 \mathrm{a}$ \\
\hline lapar 59 & $28,6 \mathrm{~dB}$ & $23,5 \mathrm{cB}$ & $23,4 \mathrm{eB}$ & $39,5 \mathrm{cA}$ & $28,8 \mathrm{f}$ \\
\hline IPR 98 & $25,6 \mathrm{~dB}$ & $25,0 \mathrm{cB}$ & $30,1 \mathrm{~dB}$ & 45,7 bA & $31,6 \mathrm{e}$ \\
\hline IPR 99 & $47,2 \mathrm{aA}$ & 30,3 bB & $41,5 \mathrm{cA}$ & $46,5 \mathrm{bA}$ & $41,4 \mathrm{~b}$ \\
\hline IPR 103 & 43,2 bB & $33,0 \mathrm{bc}$ & $46,3 \mathrm{bB}$ & $54,9 \mathrm{aA}$ & $44,3 \mathrm{a}$ \\
\hline IPR 104 & $21,0 \mathrm{eC}$ & $35,1 \mathrm{bB}$ & $25,4 \mathrm{eC}$ & $46,8 \mathrm{bA}$ & $32,1 \mathrm{e}$ \\
\hline Topázio MG 1190 & 37,4 bA & $41,7 \mathrm{aA}$ & $43,6 \mathrm{cA}$ & 43,4 bA & $41,5 \mathrm{~b}$ \\
\hline Bourbon Amarelo IAC J10 & $34,4 \mathrm{cA}$ & $19,2 \mathrm{cB}$ & $22,7 \mathrm{eB}$ & $39,3 \mathrm{cA}$ & $28,9 \mathrm{f}$ \\
\hline Média & $35,3 \mathrm{C}$ & $30,1 \mathrm{D}$ & $37,0 \mathrm{~B}$ & $43,5 \mathrm{~A}$ & 36,5 \\
\hline CV (\%) & 11,78 & & & & \\
\hline
\end{tabular}

Médias seguidas de mesmas letras minúsculas na coluna e maiúsculas na linha não diferem pelo teste de Scott-Knott com p<0,01 de probabilidade. 
et al. (2005), em trabalho de competição de cultivares resistentes à ferrugem no município de Lavras, Sul de Minas Gerais, verificaram superioridade de produção para duas cultivares do grupo Catucaí, com destaque para a cultivar Catucaí Amarelo 2SL, com produção de 69,9 sacas ha-1 ${ }^{-1}$ na segunda safra. Avaliando a resistência à ferrugem de doze novas cultivares de café desenvolvidas pelo Instituto Agronômico do Paraná, SerA et al. (2010), destacam a produtividade da cultivar IPR 103 , significativamente superior ao do padrão resistente 'Iapar 59'.

Neste trabalho, a cultivar Catucaí 785/15 propiciou desempenho inferior em relação às demais cultivares em todos os locais avaliados, com decréscimo de até 44,9\% na produtividade, em comparação com a média da melhor cultivar. O baixo desempenho em produtividade das cultivares Iapar 59 e Bourbon Amarelo IAC J10 observado no presente experimento também já foi verificado por outros autores nas regiôes Sul, Alto Paranaíba e Vale do Jequitinhonha de Minas Gerais (BотеLho et al., 2010), confirmando a baixa adaptabilidade dessas cultivares no Estado de Minas Gerais.

As maiores produtividades foram observadas no ensaio de Turmalina, cuja média foi $43,5 \mathrm{sc} \mathrm{ha}^{-1}$. Estes resultados demonstram que o ambiente influencia na expressão de parte do potencial de produção.
O vigor vegetativo está relacionado com a capacidade de adaptação das cultivares nas diferentes condiçóes edafoclimáticas em que são cultivadas. Houve diferença significativa para essa característica em todos os locais, exceto Turmalina, onde a vegetação foi semelhante entre as cultivares (Tabela 3). Em Lavras e Patrocínio, foram observados três grupos de cultivares, enquanto em Campos Altos, quatro grupos, nos quais as notas variaram de 4,5 a 8,9. As cultivares Sabiá Tardio e Pau Brasil MG1 foram as mais vigorosas nos três locais onde se identificou comportamento diferenciado entre as cultivares. É importante salientar que essas cultivares aliaram alto vigor vegetativo com elevada produtividade, bem como também se destacaram em produtividade nos referidos locais de avaliação.

Elevado vigor vegetativo correlaciona-se positivamente com a adaptaçáo da cultivar ao ambiente, refletindo em plantas com menor depauperamento (SEVERINo et al., 2002). As cultivares Catucaí 785/15 e Oeiras MG 6851 tiveram comportamento inverso, com alto grau de depauperamento, devido ao baixo vigor vegetativo em três locais (Lavras, Campos Altos e Patrocínio). Na média geral dos quatro locais, destacam-se as cultivares Acauã, Catiguá MG 1, Sacramento MG 1, Catiguá MG 2, Araponga MG 1 e Paraíso MG H419-1.

Notou-se a influência genética sobre a formação de grãos classificados em peneira alta, uma vez que foi

Tabela 3. Vigor vegetativo de 24 cultivares comerciais de cafeeiro, avaliadas nos municípios de Lavras (LA), Campos Altos (CA), Patrocínio (PA) e Turmalina (TU) em Minas Gerais, nas safras de 2008/2009 a 2011/2012 e média geral dos quatro ambientes

\begin{tabular}{|c|c|c|c|c|c|}
\hline Cultivares & LA & CA & PA & TU & Média \\
\hline Catucaí Amarelo 2 SL & $7,8 \mathrm{a}$ & $6,6 \mathrm{~d}$ & $5,9 \mathrm{~b}$ & $6,7 \mathrm{a}$ & $6,7 c$ \\
\hline Catucaí Amarelo 24/137 & $7,2 \mathrm{~b}$ & $5,9 d$ & $6,4 \mathrm{a}$ & $6,3 \mathrm{a}$ & $6,5 c$ \\
\hline Catucaí Amarelo 20/15 cv 479 & $7,3 b$ & $6,8 \mathrm{c}$ & $6,9 \mathrm{a}$ & $6,7 \mathrm{a}$ & $6,9 \mathrm{~b}$ \\
\hline Catucaí 785/15 & $6,0 \mathrm{c}$ & $5,8 d$ & $5,3 c$ & $5,9 \mathrm{a}$ & $5,9 \mathrm{c}$ \\
\hline Catucaí Vermelho 20/15 cv 476 & $7,5 \mathrm{a}$ & $6,1 \mathrm{~d}$ & $5,8 \mathrm{~b}$ & $6,4 \mathrm{a}$ & $6,3 c$ \\
\hline Sabiá Tardio & $7,8 \mathrm{a}$ & $7,5 \mathrm{~b}$ & $6,9 \mathrm{a}$ & $7,0 \mathrm{a}$ & $7,1 \mathrm{~b}$ \\
\hline IBC Palma 2 & $8,1 \mathrm{a}$ & $7,2 \mathrm{c}$ & $6,1 \mathrm{~b}$ & $6,8 \mathrm{a}$ & $7,1 \mathrm{~b}$ \\
\hline Acauã & $8,5 \mathrm{a}$ & $7,3 \mathrm{c}$ & $7,4 \mathrm{a}$ & $6,8 \mathrm{a}$ & $7,5 \mathrm{a}$ \\
\hline Oeiras MG 6851 & $6,4 c$ & $6,2 d$ & $5,3 \mathrm{c}$ & $7,5 \mathrm{a}$ & $6,2 c$ \\
\hline Catiguá MG 1 & $7,9 \mathrm{a}$ & $7,1 \mathrm{c}$ & $7,1 \mathrm{a}$ & $6,7 \mathrm{a}$ & $7,4 \mathrm{a}$ \\
\hline Sacramento MG 1 & $8,1 \mathrm{a}$ & $8,8 \mathrm{a}$ & $7,1 \mathrm{a}$ & $7,1 \mathrm{a}$ & $7,7 \mathrm{a}$ \\
\hline Catiguá MG 2 & $8,6 \mathrm{a}$ & $8,9 \mathrm{a}$ & $7,3 \mathrm{a}$ & $7,2 \mathrm{a}$ & $8,0 \mathrm{a}$ \\
\hline Araponga MG 1 & $8,1 \mathrm{a}$ & $8,0 \mathrm{~b}$ & $7,1 \mathrm{a}$ & $7,3 \mathrm{a}$ & $7,6 \mathrm{a}$ \\
\hline Paraíso MG H419-1 & $8,7 \mathrm{a}$ & $7,9 \mathrm{~b}$ & $6,5 \mathrm{a}$ & $7,1 \mathrm{a}$ & $7,5 \mathrm{a}$ \\
\hline Pau Brasil MG 1 & $7,8 \mathrm{a}$ & $7,3 \mathrm{c}$ & $6,3 \mathrm{a}$ & $6,3 \mathrm{a}$ & $6,9 b$ \\
\hline Tupi IAC 1669-33 & $7,6 \mathrm{a}$ & $6,4 \mathrm{~d}$ & $6,0 \mathrm{~b}$ & $6,8 \mathrm{a}$ & $6,7 c$ \\
\hline Obatã IAC 1669-20 & $7,3 \mathrm{~b}$ & $6,9 \mathrm{c}$ & $6,1 \mathrm{~b}$ & $6,1 \mathrm{a}$ & $6,6 \mathrm{c}$ \\
\hline lapar 59 & $7,5 \mathrm{a}$ & $6,8 \mathrm{c}$ & $4,7 \mathrm{c}$ & $6,2 \mathrm{a}$ & $6,3 c$ \\
\hline IPR 98 & $8,3 a$ & $7,3 \mathrm{c}$ & $5,9 \mathrm{~b}$ & $6,7 \mathrm{a}$ & $7,0 \mathrm{~b}$ \\
\hline IPR 99 & $7,9 \mathrm{a}$ & $6,6 \mathrm{~d}$ & $6,9 \mathrm{a}$ & $6,9 \mathrm{a}$ & $7,1 \mathrm{~b}$ \\
\hline IPR 103 & $8,2 \mathrm{a}$ & $7,3 c$ & $7,5 \mathrm{a}$ & $7,2 \mathrm{a}$ & $7,5 \mathrm{~b}$ \\
\hline IPR 104 & $7,3 b$ & $6,4 \mathrm{~d}$ & $4,5 \mathrm{c}$ & $6,4 \mathrm{a}$ & $6,2 \mathrm{c}$ \\
\hline Topázio MG 1190 & $7,0 \mathrm{~b}$ & $6,9 c$ & $4,9 c$ & $7,3 \mathrm{a}$ & $6,5 c$ \\
\hline Bourbon Amarelo IAC J10 & $6,3 c$ & $5,8 \mathrm{~d}$ & $6,5 \mathrm{a}$ & $7,3 \mathrm{a}$ & $6,5 \mathrm{c}$ \\
\hline Média & $7,6 \mathrm{~A}$ & $6,9 \mathrm{~B}$ & $6,2 \mathrm{D}$ & $6,7 \mathrm{C}$ & 6,9 \\
\hline CV(\%) & 7,6 & & & & \\
\hline
\end{tabular}

Médias seguidas de mesmas letras minúsculas na coluna e maiúsculas na linha não diferem pelo teste de Scott-Knott com p $<0,01$ de probabilidade. 
detectada diferença significativa entre as cultivares dentro de cada ambiente estudado (Tabela 4). Verifica-se que em todas as cultivares, de forma geral, as porcentagens de grãos de peneira 16 e acima foram elevadas. De acordo com o teste de Scott Knott, foi possível identificar três grupos em cada local de avaliação e quatro grupos na média dos quatro locais.

Em programas de melhoramento genético de cafeeiro, busca-se um genótipo cujo desempenho abranja, além de outras características, elevada capacidade produtiva e com maior porcentagem de grãos classificados em peneiras mais altas (FERreira et al., 2005). No presente trabalho, pode-se destacar as cultivares Catucaí Amarelo 24/137, Obatã IAC 1669-20 e Pau Brasil MG1, que, além de maior quantidade de grãos classificados em peneira alta, em todos os locais e na média dos quatro locais, também se destacaram em produtividade.

A divergência entre os grupos formados em cada local justifica o estudo da estabilidade e adaptabilidade pelo método de AnNichiarico (1992), o qual estima a probabilidade de certa progênie ter desempenho abaixo da média do ambiente (Tabela 5). O índice de confiança ( $\mathrm{I}_{\mathrm{i}}$ ) evidencia o desempenho da cultivar em relação à média do ambiente e é interferido por duas variáveis distintas, uma relacionada à produtividade e outra relativa à estabilidade, ou seja, o desvio-padrão.
No presente trabalho, algumas cultivares como Catucai Amarelo 24/137 (97,62\%), Sabiá Tardio (95,22\%), Pau Brasil MG 1 (97,71\%), Obatã IAC 1669-20 (99,36\%), IPR 103 (107,62\%) e Topázio MG 1190 (95,75\%) destacaram-se pelos maiores valores do índice de confiança (Tabela 5). Essas cultivares são promissoras porque, além da maior estabilidade nos ambientes estudados, estão entre as mais produtivas na média dos ambientes.

A cultivar IPR 103, com 90\% de confiança, alcançou índice Ii de 107,62 (índice máximo no experimento) e desvio-padrão de baixa magnitude $(10,09)$, enquanto a cultivar Bourbon Amarelo IAC J10, obteve o menor índice de confiança do ensaio $(47,49)$. Se compararmos os desempenhos dessas duas cultivares nos ambientes mais desfavoráveis para cada uma, a 'IPR 103' produzirá $44,13 \%$ a mais que a 'Bourbon Amarelo IAC 10', comparados com a média ambiental. Considerando-se a média de produtividade do Estado de Minas Gerais, em torno de 22 sacas ha $^{-1}$ (Conab, 2011), haveria um aumento de 9,71 sacas ha ${ }^{-1}$ com a escolha correta da cultivar.

As cultivares que obtiveram os menores valores de produtividade em todos os ambientes (Catucaí 785/15, Iapar 59 e Bourbon Amarelo IAC J10) tiveram também baixos índices de confiança, confirmando a baixa adaptabilidade das cultivares aos ambientes estudados (Tabelas 2 e 5).

Tabela 4. Porcentagem de grãos de peneira 16 acima de 24 cultivares comerciais de cafeeiro, avaliadas nos municípios de Lavras (LA), Campos Altos (CA), Patrocínio (PA) e Turmalina (TU) em Minas Gerais, nas safras de 2008/2009 a 2011/2012 e média geral dos quatro ambientes

\begin{tabular}{|c|c|c|c|c|c|}
\hline Cultivares & LA & CA & PA & TU & Média \\
\hline Catucaí Amarelo 2 SL & 75,1 a & $63,4 \mathrm{a}$ & $61,7 \mathrm{a}$ & 66,7 b & $66,7 \mathrm{a}$ \\
\hline Catucaí Amarelo 24/137 & $74,3 \mathrm{a}$ & $62,5 \mathrm{a}$ & $65,2 \mathrm{a}$ & $71,2 \mathrm{a}$ & $68,1 \mathrm{a}$ \\
\hline Catucaí Amarelo 20/15 cv 479 & $70,9 \mathrm{~b}$ & $50,4 \mathrm{c}$ & $51,8 \mathrm{c}$ & $60,6 \mathrm{c}$ & $58,4 \mathrm{c}$ \\
\hline Catucaí 785/15 & $69,9 \mathrm{~b}$ & $62,6 \mathrm{a}$ & $59,3 \mathrm{~b}$ & $76,8 \mathrm{a}$ & $67,2 \mathrm{a}$ \\
\hline Catucaí Vermelho 20/15 cv 476 & $69,9 \mathrm{~b}$ & $56,0 \mathrm{~b}$ & $60,8 \mathrm{~b}$ & $74,6 \mathrm{a}$ & $65,3 b$ \\
\hline Sabiá Tardio & $76,3 \mathrm{a}$ & $57,2 b$ & $56,9 \mathrm{~b}$ & $69,4 b$ & $64,9 \mathrm{~b}$ \\
\hline IBC Palma 2 & $65,3 c$ & $58,4 b$ & $56,5 b$ & $60,8 c$ & $60,3 c$ \\
\hline Acauã & $51,6 \mathrm{e}$ & $49,2 \mathrm{c}$ & $45,6 d$ & $58,2 d$ & $51,1 \mathrm{e}$ \\
\hline Oeiras MG 6851 & $66,1 c$ & $52,9 \mathrm{c}$ & $57,9 \mathrm{~b}$ & $64,6 \mathrm{c}$ & $60,4 c$ \\
\hline Catiguá MG 1 & $67,7 \mathrm{~b}$ & $63,9 \mathrm{a}$ & $47,4 d$ & $55,3 d$ & $58,6 \mathrm{c}$ \\
\hline Sacramento MG 1 & $57,2 \mathrm{~d}$ & $47,1 \mathrm{~d}$ & $44,7 d$ & $67,3 b$ & $54,0 \mathrm{~d}$ \\
\hline Catiguá MG 2 & $67,7 \mathrm{~b}$ & $46,5 d$ & $47,2 d$ & $55,9 d$ & $54,3 \mathrm{~d}$ \\
\hline Araponga MG 1 & $68,0 \mathrm{~b}$ & $58,4 b$ & $47,1 d$ & $60,8 c$ & $58,5 \mathrm{c}$ \\
\hline Paraíso MG H419-1 & $63,2 \mathrm{c}$ & $42,9 \mathrm{~d}$ & $50,4 \mathrm{c}$ & $63,3 c$ & $55,0 \mathrm{~d}$ \\
\hline Pau Brasil MG 1 & $75,2 \mathrm{a}$ & $62,5 \mathrm{a}$ & $65,5 \mathrm{a}$ & $72,2 \mathrm{a}$ & $68,7 \mathrm{a}$ \\
\hline Tupi IAC 1669-33 & $74,6 \mathrm{a}$ & $62,8 \mathrm{a}$ & $66,6 \mathrm{a}$ & $66,8 b$ & $67,7 \mathrm{a}$ \\
\hline Obatã IAC 1669-20 & $76,1 \mathrm{a}$ & $61,1 \mathrm{a}$ & $63,2 \mathrm{a}$ & $69,3 b$ & $67,5 \mathrm{a}$ \\
\hline lapar 59 & $75,2 \mathrm{a}$ & $61,5 \mathrm{a}$ & $63,1 \mathrm{a}$ & $73,0 \mathrm{a}$ & $68,2 \mathrm{a}$ \\
\hline IPR 98 & $64,4 \mathrm{c}$ & $54,4 \mathrm{c}$ & $56,0 \mathrm{~b}$ & $66,3 \mathrm{~b}$ & $60,3 c$ \\
\hline IPR 99 & $74,3 a$ & $65,6 \mathrm{a}$ & $59,3 b$ & $69,7 \mathrm{~b}$ & $67,3 \mathrm{c}$ \\
\hline IPR 103 & 73,9 a & $59,1 \mathrm{~b}$ & $67,8 \mathrm{a}$ & 69,4 b & $67,6 \mathrm{a}$ \\
\hline IPR 104 & $74,3 \mathrm{a}$ & $52,9 \mathrm{c}$ & $62,7 \mathrm{a}$ & $74,3 \mathrm{a}$ & $66,1 \mathrm{a}$ \\
\hline Topázio MG 1190 & $68,7 \mathrm{~b}$ & $59,8 b$ & $51,9 \mathrm{c}$ & $69,8 b$ & $62,6 \mathrm{~b}$ \\
\hline Bourbon Amarelo IAC J10 & $71,9 \mathrm{~b}$ & $66,7 \mathrm{a}$ & $51,3 \mathrm{c}$ & $67,1 \mathrm{~b}$ & $64,2 b$ \\
\hline Média & $69,6 \mathrm{~A}$ & $57,4 \mathrm{C}$ & $56,6 \mathrm{C}$ & $66,8 \mathrm{~B}$ & 62,6 \\
\hline CV(\%) & 7,18 & & & & \\
\hline
\end{tabular}

Médias seguidas de mesmas letras minúsculas na coluna e maiúsculas na linha não diferem pelo teste de Scott-Knott com p<0,01 de probabilidade. 
Tabela 5. Estimativa da média (Yi), dos desvios-padrão ( $\mathrm{Si}$ ) e do índice de confiança (Ii), segundo o método proposto por AnnicChiarico (1992), para produtividade de café beneficiado, em sacas de $60 \mathrm{~kg}$, de 24 cultivares comerciais de cafeeiro avaliadas nas safras de 2008/2009 a 2011/2012, em experimentos desenvolvidos nas regióes Sul, Alto Paranaíba e Vale do Jequitinhonha, no Estado de Minas Gerais

\begin{tabular}{lrrr}
\hline Cultivares & \multicolumn{1}{c}{ Yi } & \multicolumn{1}{c}{ Si } & \multicolumn{1}{c}{ li* $^{*}$} \\
\hline Catucaí Amarelo 2 SL & 106,68 & 16,92 & 84,99 \\
\hline Catucaí Amarelo 24/137 & 118,55 & 16,32 & 97,62 \\
\hline Catucaí Amarelo 20/15 cv 479 & 106,06 & 14,67 & 87,25 \\
\hline Catucaí 785/15 & 71,22 & 12,93 & 54,65 \\
\hline Catucaí Vermelho 20/15 cv 476 & 82,54 & 13,33 & 65,45 \\
\hline Sabiá Tardio & 126,15 & 24,14 & 95,22 \\
\hline IBC Palma 2 & 112,87 & 17,83 & 90,01 \\
\hline Acauã & 98,17 & 20,08 & 72,43 \\
\hline Oeiras MG 6851 & 91,48 & 15,18 & 72,02 \\
\hline Catiguá MG 1 & 85,11 & 15,75 & 64,92 \\
\hline Sacramento MG 1 & 95,91 & 19,19 & 71,31 \\
\hline Catiguá MG 2 & 98,97 & 26,97 & 64,40 \\
\hline Araponga MG1 & 91,58 & 10,69 & 77,87 \\
\hline Paraíso MG H419-1 & 92,10 & 21,10 & 65,05 \\
\hline Pau Brasil MG 1 & 118,26 & 16,03 & 97,71 \\
\hline Tupi IAC 1669-33 & 100,30 & 22,00 & 72,10 \\
\hline Obatã IAC 1669-20 & 124,10 & 19,30 & 99,36 \\
\hline lapar 59 & 78,29 & 14,90 & 59,20 \\
\hline IPR 98 & 84,41 & 13,99 & 66,47 \\
\hline IPR 99 & 111,94 & 22,37 & 83,26 \\
\hline IPR 103 & 120,56 & 10,09 & 107,62 \\
\hline IPR 104 & 87,30 & 25,60 & 54,49 \\
\hline Topázio MG 1190 & 116,81 & 16,43 & 95,75 \\
\hline Bourbon Amarelo IAC J10 & 80,53 & 25,77 & 47,49 \\
\hline *Nível de significância adotado =0,1. & & & \\
\hline
\end{tabular}

\section{CONCLUSÃO}

As cultivares Sabiá Tardio, Pau Brasil MG1, Obatã IAC 1669-20, Catucaí Amarelo 24/137 e IPR 103 são promissoras para o cultivo nas regióes. Essas cultivares proporcionam estabilidade e adaptabilidade independentemente do ambiente, com desempenho agronômico destacado.

\section{AGRADECIMENTOS}

Os autores agradecem ao Consórcio Brasileiro de Pesquisa e Desenvolvimento do Café (CBPD-Café) e ao Instituto Nacional de Ciência e Tecnologia do Café (INCT Cafél $\mathrm{CNPq}$ ) pelo apoio financeiro ao projeto e ao $\mathrm{CNPq}$ pela concessão das bolsas PNPD (AMC e JCR).

\section{REFERÊNCIAS}

ANNICCHIARICO, P. Cultivar adaptation and recommendation from alfafa trials in Northern Italy. Journal Genetic and Breeding, v.46, p.269-278, 1992.
BONOMO, P.; CRUZ, C.D.; VIANA, J.M.S.; PEREIRA, A.A.; OLIVEIRA,V.R.; CARNEIRO, P.C.S. Avaliação de progênies obtidas de cruzamentos de descendentes do Híbrido de Timor com as cultivares Catuaí Vermelho e Catuaí Amarelo. Bragantia, v.63, p.207-219, 2004.

BOTELHO, C.E.; MENDES, A.N.G.; CARVALHO, G.R.; BARTHOLO, G.F.; CARVALHO, S.P. Seleção de progênies F4 de cafeeiro obtidas pelo cruzamento de Icatu com Catimor. Revista Ceres, v.57, p.274-281, 2010.

CARGNIN, A.; SOUZA, M.A.; CARNEIRO, P.C.S.; SOFIATTI, $\mathrm{V}$. Interação entre genótipos e ambientes e implicaçóes em ganhos com seleção em trigo. Pesquisa Agropecuária Brasileira, v.41, p.987993, 2006.

CARVALHO, A.; MÔNACO, L.C.; FAZUOLI, L.C. melhoramento do café XL - Estudos de progênies e híbridos de café Catuaí. Bragantia, v.38, p.202-216, 1979.

CARVALHO, G.R.; BOTELHO, C.E.; BARTHOLO, G.F; PEREIRA, A.A.; NOGUEIRA, A.M.; CARVALHO, A.M. Comportamento de progênies F4 x obtidas por cruzamento de Icatu com Catimor. Ciência e Agrotecnologia, v.33, p.47-52, 2009.

CILAS, C.; MONTAGNON, C.; BAR-HEN, A. Yield stability in clones of Coffea canephora in the short and medium term: longitudinal data analyses and measures of stability over time. Tree Genetics and Genome, v.7, p.421-429, 2011.

CORRÊA, L.V.T.; MENDES, A.N.G.; BARTHOLO, G.F. Comportamento de cafeeiro Icatu. Ciência e Agrotecnologia, v.30, p.618-622, 2006.

CONAB. Acompanhamento da Safra Brasileira Café Safra 2011. Segunda estimativa, maio/2011/ Companhia Nacional de Abastecimento. Brasília: Conab, 2011. Disponível em: http://www. conab.gov.br/OlalaCMS/uploads/arquivos/11_05_10_09_04_16_ boletim_ccafe_portugues_-_maio_-_2011_2o_lev..pdf. Acesso em $28 / 4 / 2012$

CONDÉ, A.B.T.; COELHO, M.A.; YAMANAKA, C.H.; CORTE, H.R. Adaptabilidade e estabilidade de genótipos de trigo sob cultivo de sequeiro em Minas Gerais. Pesquisa Agropecuária Tropical, v.40, p.45-52, 2010.

CUCOLOTTO, M.; PIPOLO, V.C.; GARBUGLIO, D.D.; FONSECA JUNIOR, N.S.; DESTRO, D.; KAMIKOGA, M.K. Genotype $x$ environment interaction in soybean: evaluation through three methodologies. Crop Breeding and Applied Biotechnology, v.7, p.270-277, 2007.

DIAS, F.P.; MENDES, A.N.G.; SOUZA, C.A.S.; CARVALHO, S.P.; BOTELHO, C.E.; RASO, B.S.M. Caracterização de progênies de cafeeiro (Coffea arabica L.) selecionadas em Minas Gerais: I caracteres relacionados ao crescimento vegetativo. Revista Ceres, v. 52, p.73-83, 2005.

FERREIRA, D.F.; ZAMBALDE, A.L. Simplificação de algumas técnicas especiais da experimentaçáo agropecuária no MAPGEN e softwares correlatos. In: CONGRESSO DA SOCIEDADE BRASILEIRA DE INFORMÁTICA, 1997, Belo Horizonte. Anais... Belo Horizonte: Sociedade Brasileira de Informática, 1997. p.285-291. 
FERREIRA, A.; CECON, P.R.; CRUZ, C.D.; FERRÃO, R.G.; SILVA, M.F.; FONSECA, A.F.A.; FERRÃO, M.A.G. Seleção simultânea de Coffea canephora por meio da combinaçáo de análise de fatores e índices de seleção. Pesquisa Agropecuária Brasileira, v.40, p.1189-1195, 2005.

FERREIRA, D.F. Sisvar: a computer statistical analysis system. Ciência e Agrotecnologia, v.35, p.1039-1042, 2011.

GICHIMU, B.M.; OMONDI, C.O. Early performance of five newly developed lines of Arabica coffee under varying environment and spacing in Kenya. Agriculture and Biology Journal of North America, v.1, p.32-39, 2010.

GRUNVALD, A. K.; CARVALHO, C.G.P.; OLIVEIRA, A.C.B.; ANDRADE, C.A.B. Adaptabilidade e estabilidade de genótipos de girassol no Brasil Central. Pesquisa Agropecuária Brasileira, v.43, p.1483-1493, 2008.

PAIVA, R.N.; CARVALHO, C.H.S.; MENDES, A.N.G.; ALMEIDA, S.R.; MATIELLO, J.B.; FERREIRA, R.A.
Comportamento agronômico de progênies de cafeeiro (Coffea arabica L.) em Varginha-MG. Coffee Science, v.5, p.49-58, 2010.

SANTOS, H.G.; JACOMINE, P.K.T.; ANJOS, L.H.C.; OLIVEIRA, V.A.; OLIVEIRA, J.B.; COELHO, M.R.; LUMBRERAS, J.F.; CUNHA, T.J.F. (Ed.). Sistema brasileiro de classificação de solos. Rio de Janeiro: Embrapa Solos, 2006. 306p.

SERA, G.H.; SERA, T.; FONSECA, I.C.B.; ITO, D.S. Resistência à ferrugem alaranjada em cultivares de café. Coffee Science, v.5, p. 59-66, 2010.

SEVERINO, L.S.; SAKIYAMA, N.S.; PEREIRA, A.A.; MIRANDA, G.V.; ZAMBOLIM, L.; BARROS, U.V. Associaçôes da produtividade com outras características agronômicas de café (Coffea arabica L. Catimor). Acta Scientiarum Agronomy, v.24, p.1467-1471, 2002.

VASCONCELOS, E.S.; REIS, M.S.; CRUZ, C.D.; SEDIYAMA, T.; SCAPIM, C.A. Adaptability and stability of semilate and late maturing soybean genotypes in Minas Gerais state. Acta Scientiarum Agronomy, v.32, p.411-415, 2010. 